\title{
The New Look Nephron
}

Why bother to change the editorial policy of a journal like Nephron, old established as nephrology journals go, and with a small but faithful group of readers? We feel that Nephron, the old style Nephron, was a journal that catered for the tastes of a small group of renal physiologists in the main, and that clinical nephrological problems received relatively scant attention. As clinical nephrologists we felt that a 'new look' Nephron was necessary, and this is what we intend to provide for you. We aim to give you a journal with a fair mixture of clinical nephrological and pathological studies with adequate representation of renal physiology, radio isotope work, and dialysis and transplant problems. We believe that 'new look' Nephron should have editorials, review articles, as well as original work, from time to time. About 2 or 3 times a year we intend to publish entire issues devoted to symposia on subjects of interest to nephrologists, including current problems of dialysis, in divalent ion metabolism, paediatric nephrological topical issues, uraemic toxins, hypertension, renin metabolism, glomerulonephritis, immunological problems in transplantation. These symposia will have guest editors who are leaders in their field. We intend to have a consumer reports - unbiased because of our lack of dependence on advertisers - on artificial kidneys, coils, and all the paraphenalia which plague our lives because there is at present little up-to-date information on the subject which is unbiased. We would solicit short reports of this type.

You will notice we are publishing only in English. We flatter ourselves that neither French or German are as widely read as English, and the fact of using one single language saves space. It will obviously take some time to change the style of Nephron to the correct mixture of clinical nephrology and physiology. In this we rely

2

\section{Editorial}

heavily on our nephrological colleagues - you, the readers, to help us. We are using two wellknown referees per article and will not tolerate rubbish under a clinical or other guise. We will be happy to publish the proceedings of national societies of nephrology free of charge in abstract form.

We see no clash of interests with Kidney International which would appear to aim to provide for the interests of renal physiology more than the 'new look' Nephron. We wish the International Society of Nephrology well with its new venture. We believe that Kidney International and the 'new look' Nephron fill different needs and that most nephrologists will buy both.

We will aim to publish within 3 months of receipt of articles, so overcoming the problem of too great a delay. We are utilising a Swiss Editorial address so that the joint editors-in-chief can get the papers sent on simultaneously, whatever the international situation. We welcome readers' letters - they increase the standard of articles published.

We are encouraged by our very distinguished editorial board and feel assured of the success of our venture with you - a 'new look' Nephron.

G. M. Berlyne, Beersheva, Israel S. Giovannetti, Pisa, Italy 